ARTICLE

\title{
Tuning lithium-peroxide formation and decomposition routes with single-atom catalysts for lithium-oxygen batteries
}

Li-Na Song1,7, Wei Zhang ${ }^{2,3,7}$, Ying Wang 4,7, Xin Ge², Lian-Chun Zou1', Huan-Feng Wang ${ }^{1}$, Xiao-Xue Wang ${ }^{1}$, Qing-Chao Liu' ${ }^{5}$ Fei $\mathrm{Li}^{1} \&$ Ji-Jing Xu (1) ${ }^{1,6 \times}$

Lithium-oxygen batteries with ultrahigh energy density have received considerable attention as of the future energy storage technologies. The development of effective electrocatalysts and a corresponding working mechanism during cycling are critically important for lithiumoxygen batteries. Here, a single cobalt atom electrocatalyst is synthesized for lithium-oxygen batteries by a polymer encapsulation strategy. The isolated moieties of single atom catalysts can effectively regulate the distribution of active sites to form micrometre-sized flower-like lithium peroxide and promote the decomposition of lithium peroxide by a one-electron pathway. The battery with single cobalt atoms can operate with high round-trip efficiency (86.2\%) and long-term stability (218 days), which is superior to a commercial $5 \mathrm{wt} \%$ platinum/carbon catalyst. We reveal that the synergy between a single atom and the support endows the catalyst with excellent stability and durability. The promising results provide insights into the design of highly efficient catalysts for lithium-oxygen batteries and greatly expand the scope of future investigation.

\footnotetext{
${ }^{1}$ State Key Laboratory of Inorganic Synthesis and Preparative Chemistry, College of Chemistry, Jilin University, 130012 Changchun, PR China. ${ }^{2}$ Electron Microscopy Center, School of Materlals Science and Engneering, Jilin University, 130012 Changchun, PR China. ${ }^{3}$ IKERBASQUE, Basque Foundation for Science, 48013 Bilbao, Spain. ${ }^{4}$ State Key Laboratory of Rare Earth Resource Utilization, Changchun Institute of Applied Chemistry Chinese Academy of Sciences, 130022 Changchun, PR China. ${ }^{5}$ College of Chemistry and Molecular Engineering, Zhengzhou University, 100 Science Road, 450001 Zhengzhou, PR China. ${ }^{6}$ International Center of Future Science, Jilin University, 130012 Changchun, PR China. ${ }^{7}$ These authors contributed equally: Li-Na Song, Wei Zhang, Ying Wang. ${ }^{凶}$ email: jijingxu@jlu.edu.cn
} 
R echargeable lithium-oxygen $\left(\mathrm{Li}-\mathrm{O}_{2}\right)$ batteries based on the reversible formation and decomposition of $\mathrm{Li}_{2} \mathrm{O}_{2}$ provide a theoretical specific energy density ( $3500 \mathrm{Wh} \mathrm{kg}^{-1}$ ) that is 5-10 times higher than that of conventional Li-ion batteries, thus showing appreciable potential for vehicle applications ${ }^{1-6}$. A typical nonaqueous $\mathrm{Li}-\mathrm{O}_{2}$ battery consists of a $\mathrm{Li}$-metal anode, $\mathrm{Li}^{+}$-conducting electrolyte, and porous oxygen cathode. During discharge, $\mathrm{O}_{2}$ is preferentially reduced and then combines with $\mathrm{Li}^{+}$to form $\mathrm{Li}_{2} \mathrm{O}_{2}$ (the oxygen reduction reaction, ORR). The $\mathrm{Li}_{2} \mathrm{O}_{2}$ is subsequently converted back into $\mathrm{Li}$ and $\mathrm{O}_{2}$ during charging (the oxygen evolution reaction, OER). The electrocatalyst can effectively enhance the kinetics of both the ORR and OER, further improving the round-trip efficiency, which is crucial to energy storage equipment. In response, extensive research efforts have been made to develop highly active catalysts to boost the ORR and OER kinetics, such as the use of carbonaceous materials $^{7-9}$, transition-metal oxides ${ }^{10-12}$, noble metals ${ }^{13-15}$, and perovskites ${ }^{16-18}$. In fact, most of the aforementioned catalysts can be widely used to reduce the large overpotential; however, they have severe, unavoidable shortcomings, such as their high cost and scarcity along with their poor durability and relatively low capacity. Therefore, developing highly active and stable nonprecious metal catalysts for $\mathrm{Li}-\mathrm{O}_{2}$ batteries is significantly important. Moreover, the deposition/decomposition route of the $\mathrm{Li}_{2} \mathrm{O}_{2}$ discharge products is also an important factor in determining the kinetics of the ORR and OER reactions. Many different models for the $\mathrm{Li}_{2} \mathrm{O}_{2}$ growth mechanism have been proposed, such as a surface-adsorption pathway and a solvationmediated pathway, which are controlled by the $\mathrm{DN}$ value of the electrolyte ${ }^{19}$, adsorption energy of the cathode to intermediate species $^{5}$, cluster size ${ }^{20}$ and facet engineering of the catalyst ${ }^{21}$. All mentioned studies suggest that the solubility of $\mathrm{LiO}_{2}$ intermediates in the electrolyte determines the deposition behaviour of $\mathrm{Li}_{2} \mathrm{O}_{2}$. However, little is known about the effective decomposition mechanism after recharging by an enhanced solvation-mediated pathway, which becomes a large hindrance of the round-trip efficiency and cycle life of the battery. As a result, adopting an effective electrocatalyst to discuss in detail the mechanism of $\mathrm{O}_{2}$ reduction to $\mathrm{Li}_{2} \mathrm{O}_{2}$ while discharging and the reverse process while charging will be an another way to design high round-trip efficiency in $\mathrm{Li}-\mathrm{O}_{2}$ batteries.

Heterogeneous single-atom catalysts (SACs) referring to atomically dispersed active metal centres on a support have attracted considerable interest in recent years. The attention is because of the special electronic structure and maximized atomic utilization of such materials, which differ greatly from those of conventional nano- or subnano-counterparts ${ }^{22-24}$. Moreover, the single-atom nature of the active centres in SACs and the resulting low-coordination environment and the enhanced metal-support interactions all provide robust catalytic performance in a number of heterogeneous reactions, such as the carbon dioxide reduction ${ }^{24,25}$, hydrogen evolution reaction ${ }^{26,27}$ and photocatalytic reactions 28,29 . In principle, it is vital to explore the electrochemistry and catalytic mechanism when SACs are applied in $\mathrm{Li}-\mathrm{O}_{2}$ batteries.

With this in mind, to the best of our knowledge, for the first time, we propose a strategy of using a single-atom material as a cathode catalyst for $\mathrm{Li}-\mathrm{O}_{2}$ batteries and develop a strategy inspired by the growth process of strawberries by combining polymer encapsulation with template replication to fabricate a hollow N-doped carbon sphere with isolated single Co sites $(\mathrm{N}$ HP-Co SACs). The atomically dispersed cobalt sites are used to support the ORR and OER reactions. The hollow substrate is used to support the fast mass transfer of electrons, electrolytes, and $\mathrm{O}_{2}$. During the discharge process, the homogeneous atomic sites serve as nucleation sites for the growth of isolated nanosheet-like $\mathrm{Li}_{2} \mathrm{O}_{2}$, thus favouring subsequent decomposition during charging. More $\mathrm{LiO}_{2}$ intermediates form in the electrolyte by the SACs during charging, which can promote $\mathrm{Li}_{2} \mathrm{O}_{2}$ decomposition through a one-electron process. Benefiting from these merits, $\mathrm{Li}-\mathrm{O}_{2}$ batteries with these N-HP-Co SACs exhibit relatively low overpotential, high specific capacity, and superior rate capability. Moreover, their by-products can be effectively consumed with the help of SACs while displaying excellent cycling stability.

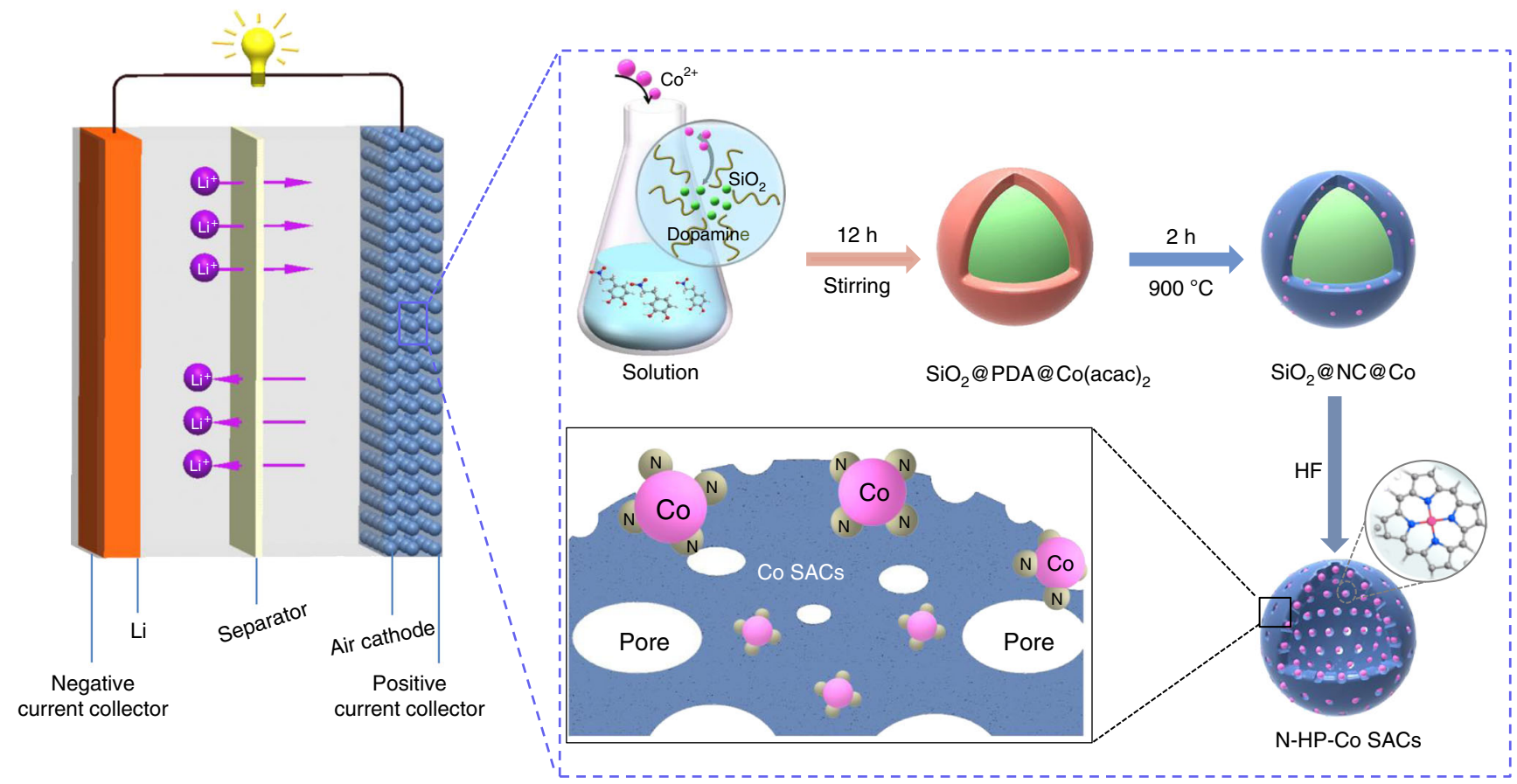

Fig. 1 Schematic illustration showing the synthesis of N-HP-Co SACs. The composition in the cell (left) and the synthesis procedures for the N-HP-Co SACs (right). 
a
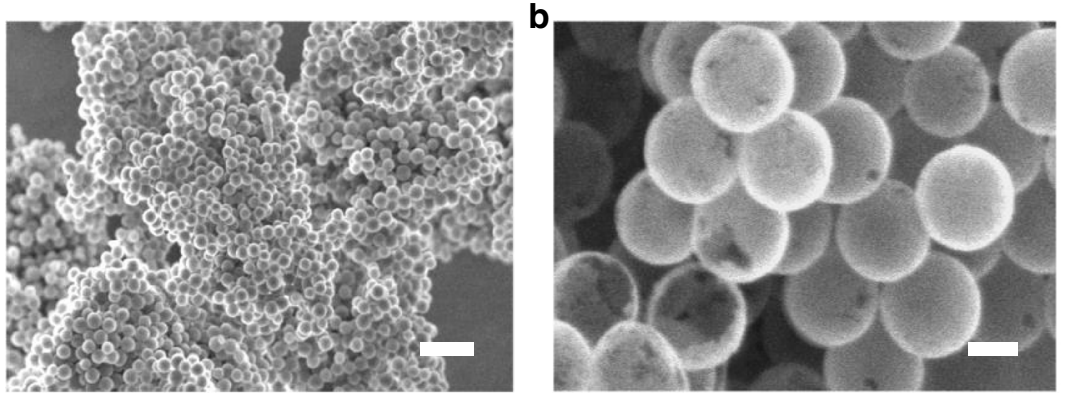

d
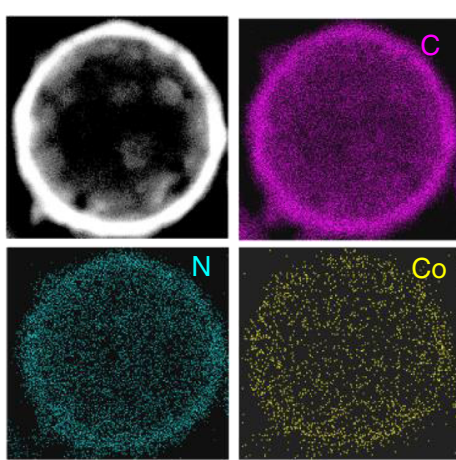

\section{g}

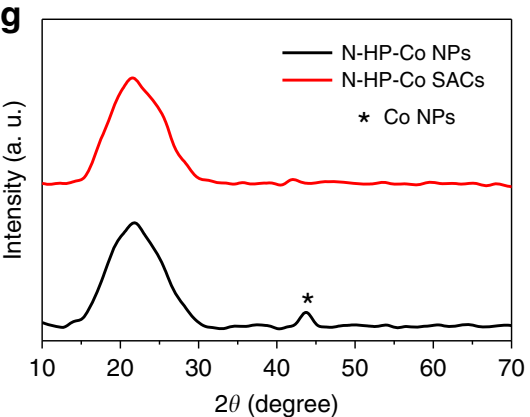

e

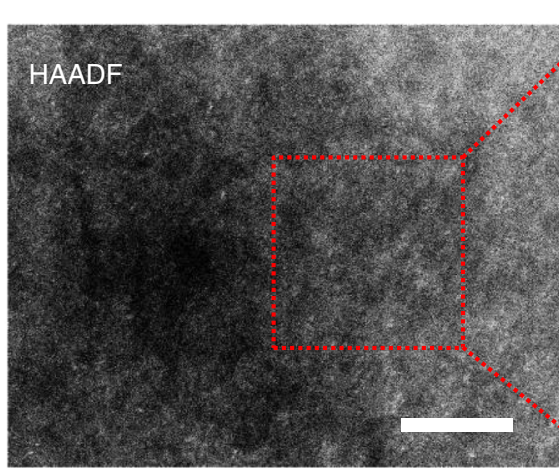

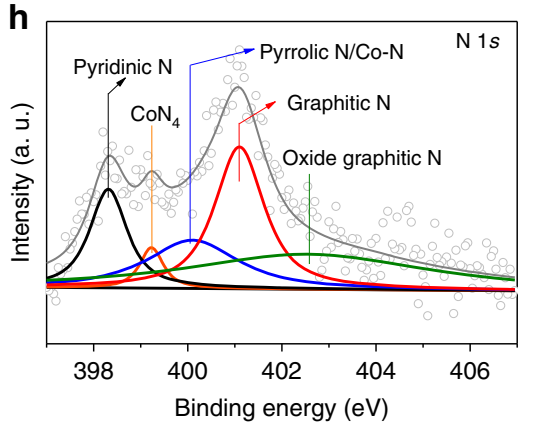
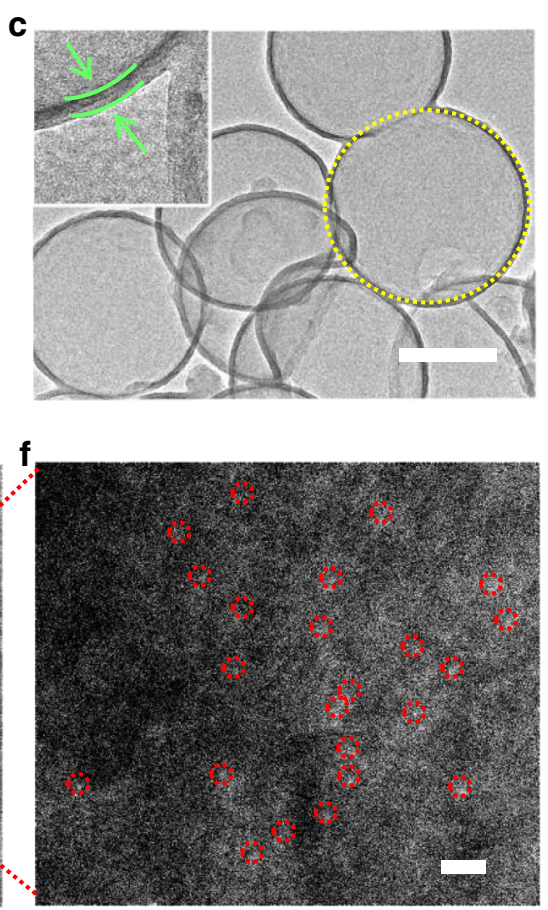

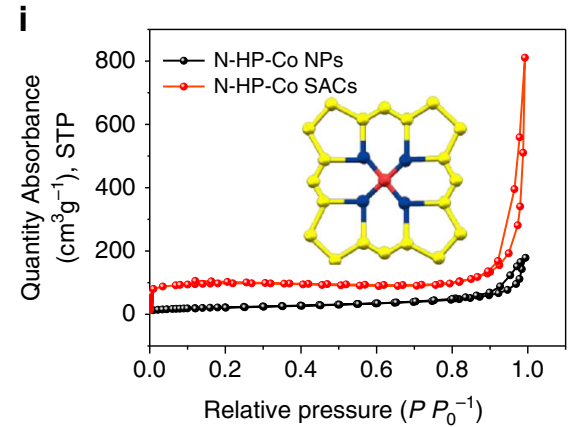

Fig. 2 Characterization of N-HP-Co SACs. SEM images of N-HP-Co SACs, $1 \mu \mathrm{m}$ in (a), $200 \mathrm{~nm}$ in (b); c TEM image of N-HP-Co SACs. d HAADF-STEM image, $50 \mathrm{~nm}$ in (d); and the corresponding elemental maps. e HAADF-STEM image, $5 \mathrm{~nm}$ in (e), f enlarged image of N-HP-Co SACs, 2 nm in (f); $\mathbf{g}$ PXRD graphs of N-HP-Co NPs and Co-SACs. h High-resolution N 1s XPS spectra of N-HP-Co SACs. i N 2 adsorption-desorption isotherms of N-HP-Co NPs and Co SACs at $77 \mathrm{~K}$. BET: $80.5 \mathrm{~cm}^{3} \mathrm{~g}^{-1}$ with N-HP-Co NPs; $312.3 \mathrm{~cm}^{3} \mathrm{~g}^{-1}$ with N-HP-Co SACs.

\section{Results}

Synthesis and structure of N-HP-Co SACs. The synthesis procedures for the hollow $\mathrm{N}$-doped porous carbon sphere-structured atomically dispersed Co-N-C catalysts are depicted in Fig. 1. Inspired by an ingenious growth process in nature, we herein demonstrate the "aggregate fruit" electrocatalyst design for $\mathrm{Li}-\mathrm{O}_{2}$ batteries. First, a spherical silica template with a particle size of $\sim 400 \mathrm{~nm}$ (Supplementary Fig. 1a) was prepared by the Stöber method $^{30}$. The cobalt complexes are mixed with dopamine monomers, which polymerize on the silica spheres to form $\mathrm{SiO}_{2} @ \mathrm{PDA} @ \mathrm{Co}(\mathrm{acac})_{2}$ nanospheres (Supplementary Fig. 1b). Then, SiO $@ @$ N-doped carbon@Co porous carbon spheres are obtained by pyrolysis to convert the coated poly-dopamine into carbon, followed by etching the silica core with HF; thus, nitrogen-hollow-porous-Co single-atom catalysts (N-HP-Co SACs) can be successfully obtained. During the polymerization process, the single Co atom is the "seed", which grows into the fruitlet, while the carbon is the "fruit", thus forming the "aggregate fruit". This special structure provides more stable Co SACs owing to strong metal-support interactions.

Scanning electron microscopy (SEM) and transmission electron microscopy (TEM) imaging show that the N-HP-Co
SAC samples consist of nanospheres with uniform diameters of $\sim 400 \mathrm{~nm}$, and the coating thickness is $\sim 10 \mathrm{~nm}$ (Fig. 2a-c). However, the existence and atomic distribution of Co elements are identified via the high-angle annular dark field scanning transmission electron microscopy (HAADF-STEM) image and corresponding EDX spectra for the composite materials. Figure $2 \mathrm{~d}$ demonstrates that $\mathrm{C}$ and $\mathrm{N}$ are homogeneously distributed on the carbon, while no Co nanoparticles (Co NPs) are observed. Numerous uniformly distributed white bright dots at the atomic scale are detected on the surface of the carbon spheres, which are assigned to the Co atoms (Fig. 2e, f). In contrast, the image of Co NPs with a size of $\sim 10 \mathrm{~nm}$ can be clearly observed through TEM (Supplementary Fig. 2). The Co content of the sample is found to be $0.6 \mathrm{wt} \%$ by the EDX spectra (Supplementary Fig. 3), which is nearly in accordance with the value of $0.56 \mathrm{wt} \%$ by inductively coupled plasma optical emission spectroscopy (Supplementary Table 1). As presented in Fig. 1g, the X-ray diffraction (XRD) pattern of the N-HP-Co NPs displays a sharp peak that can be indexed to the crystal surface of metallic cobalt. No other peaks related to metallic Co or other cobalt compounds are observed in the N-HP-Co sample, further demonstrating the single-atom nature of the Co. Notably, all the 

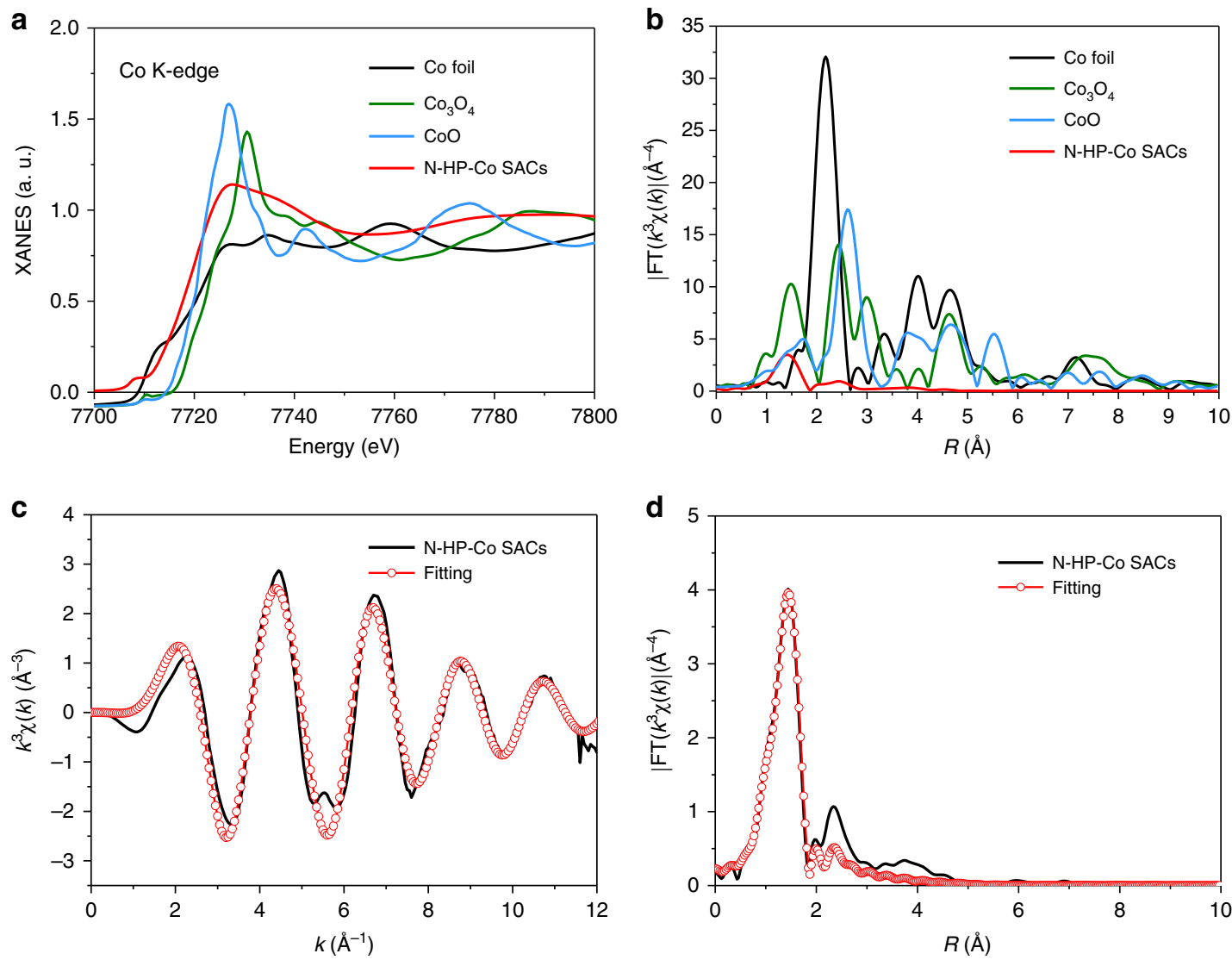

Fig. 3 Atomic structural analysis. a XANES spectra and $\mathbf{b}$ Fourier transform of the Co K-edge of N-HP-Co SACs $\mathrm{Co}_{3} \mathrm{O}_{4}, \mathrm{CoO}_{0}$, and $\mathrm{Co}$ foil. c, $\mathbf{d}$ The corresponding EXAFS fitting curves of N-HP-Co SACs at the $k$ space and $R$ space, respectively.

samples exhibit similar carbon structures with dominant D and $\mathrm{G}$ bands at 1350 and $1585 \mathrm{~cm}^{-1}$ (Supplementary Fig. 4), which are associated with the disordered carbons and $s p^{2}$ hybridized graphitic carbons, respectively ${ }^{31}$. Remarkably, the N-HP-Co SACs demonstrate a relatively high $I_{\mathrm{D}} / I_{\mathrm{G}}$ value of 0.91 , suggesting that they have the largest number of defects caused by the Co atoms in the carbon spheres, which can act as active sites for subsequent reactions. X-ray photoelectron spectroscopy (XPS) was conducted to investigate the binding states of Co, $\mathrm{N}$ and $\mathrm{C}$ in the N-HP-Co SAC catalysts, as shown in Fig. 2h. XPS presents the peaks assigned to $\mathrm{Co}, \mathrm{N}, \mathrm{O}$ and $\mathrm{C}$ in the survey spectrum of the N-HP-Co SACs, illustrating the existence of Co and $\mathrm{N}$ in the graphitic carbon framework (Supplementary Fig. 5a). The high-resolution XPS spectrum of $\mathrm{N} 1 s$ can be divided into five peaks, which correspond to pyridinic-N, $\mathrm{CoN}_{4}$, pyrrolic-N/Co-N, graphitic-N and oxide graphitic-N. In addition, pyridinic- $\mathrm{N}$ provides coordination sites to atomic $\mathrm{Co}$ in the form of $\mathrm{Co}-\mathrm{N}$, and graphitic- $\mathrm{N}$ affects the geometric and electronic structure of the carbon skeleton; graphitic- $\mathrm{N}$ groups also reveal a positive function in improving the limiting current density of the catalyst towards ORR ${ }^{32}$. Furthermore, the peak at $285.6 \mathrm{eV}$, which is assigned to the carbon atom bonding with $\mathrm{N}$ in the C $1 s$ spectrum (Supplementary Fig. 5b), further confirms the formation of $\mathrm{N}$-doped graphitic carbon. Specifically, compared with the content of $s p^{2} \mathrm{C}$, the as-prepared N-HP-Co SACs contain much less $s p^{3} \mathrm{C}$, indicating enhanced electroconductivity ${ }^{33}$. The above characteristics indicate that in the $\mathrm{N}$ HP-Co SACs, Co is atomically dispersed in the N-doped hollow porous carbon matrix, and a Co atom may coordinate with four $\mathrm{N}$ atoms around it, i.e., the formation of a $\mathrm{CoN}_{4}$ moiety. Importantly, the N-HP-Co SACs possess a large BET-specific surface area of $312.3 \mathrm{~cm}^{3} \mathrm{~g}^{-1}$ and pore volume of $1.3 \mathrm{~cm}^{3} \mathrm{~g}^{-1}$ (Fig. 2i, Supplementary Fig. 6), which provide channels for fast mass transport of oxygen and electrolytes and exposure of the abundant active sites.

As shown in Fig. 3, the local chemical bonding state of the Co atoms in the N-HP-Co SACs was further verified by X-ray absorption fine structure (XAFS) measurements. The Co K-edge absorption near-edge structure (XANES) spectra of the N-HP-Co SACs were obtained, as well as those of $\mathrm{Co}$ foil, $\mathrm{Co}_{3} \mathrm{O}_{4}$, and $\mathrm{CoO}$ to be used for comparison (Fig. 3a). In detail, the near-edge absorption energy of the N-HP-Co SACs is suited between that of the Co foil and $\mathrm{CoO}$, suggesting that the isolated $\mathrm{Co}$ atoms bear a positive charge between the metal cobalt with zerovalent Co (0) and $\mathrm{CoO}$ with bivalent $\mathrm{Co}$ (II); the above result indicates the $\mathrm{N}$ coordinated chemical state of the single Co atoms. Compared with the Co foil, the Fourier transform (FT) $\mathrm{k}^{3}$-weighted extended $\mathrm{X}$-ray absorption fine structure (EXAFS) spectrum of the N-HPCo SACs shows no appreciable Co-Co coordination peak or other high shell peaks. As expected, the FT EXAFS spectrum of N-HP-Co SACs displays a strong peak at $1.4 \AA$, demonstrating the main peak belonging to the Co-N scattering path (Fig. 3b). Furthermore, the coordination number of the centre Co atom is $\sim 4$ according to the EXAFS fitting (Fig. 3c, d, Supplementary Fig. 7), and the mean Co-N/C bond length is $1.96 \AA$ (Supplementary Table 2), further indicating the formation of a $\mathrm{CoN}_{4}$ moiety.

Discharge behaviour with the N-HP-Co SAC catalysts. The electrochemical behaviour evaluated in the $\mathrm{Li}-\mathrm{O}_{2}$ batteries is shown in Fig. 4. For comparison, a $0.6 \%$ commercial Pt/C was 

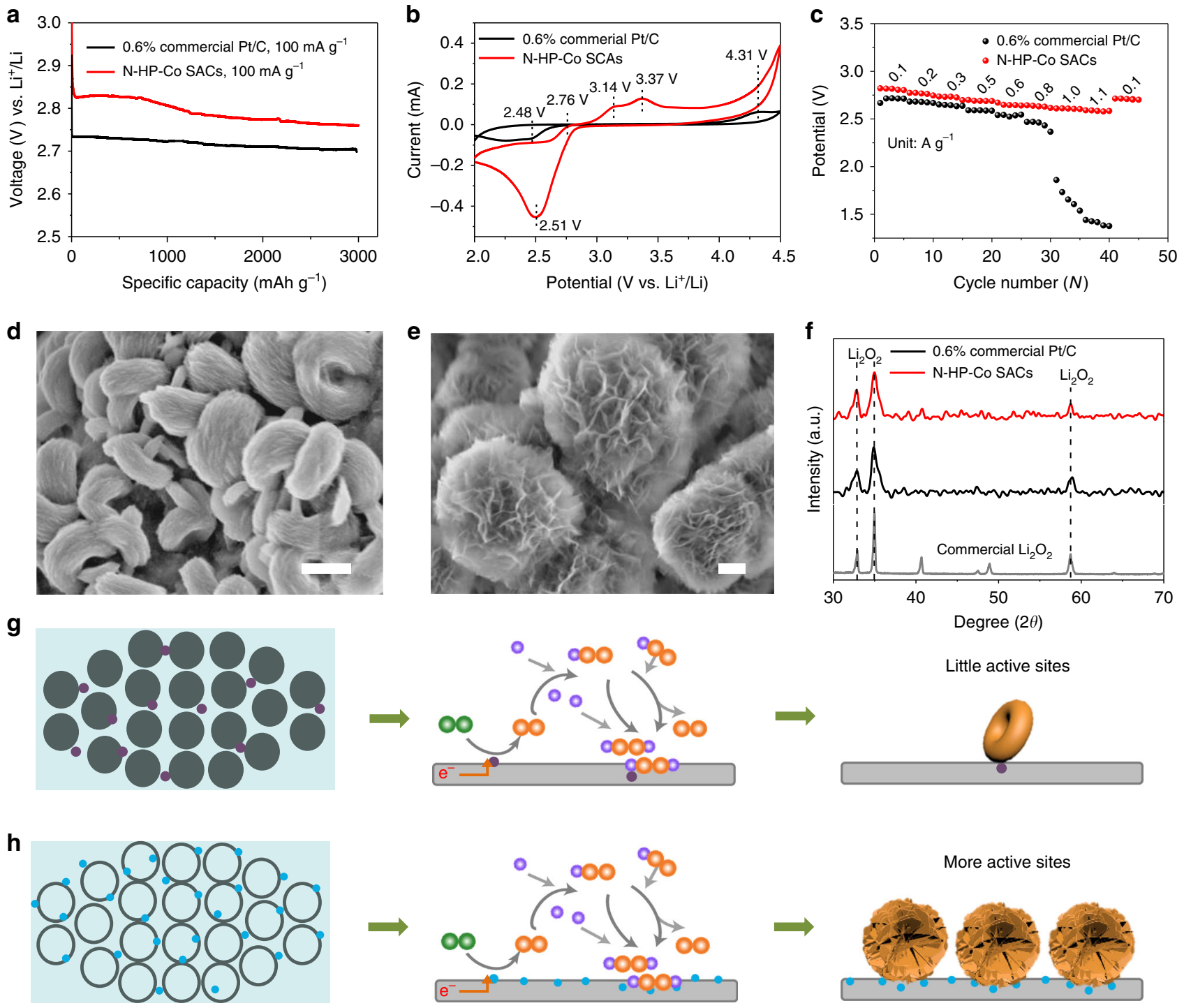

$$
\text { Carbon }
$$

$$
\text { Pt catalyst }
$$

- Co SACs

$\infty \mathrm{O}_{2}$

$\mathrm{OO}_{2}^{-}$

O Solvated Li+

$000 \mathrm{LiO}_{2}$

$0000 \mathrm{Li}_{2} \mathrm{O}_{2}$

Fig. 4 Discharge performance and characterization of discharge products. a First discharge curves with $0.6 \%$ commercial Pt/C and N-HP-Co SACs at a current density of $100 \mathrm{~mA} \mathrm{~g}^{-1}$ with a limiting specific capacity of $3000 \mathrm{mAh} \mathrm{g}^{-1}$. b CV curves. c Full range rate performances of $\mathrm{Li}_{-} \mathrm{O}_{2}$ batteries at different current densities. d, e FESEM images of the discharged cathodes with (d), $0.6 \%$ commercial Pt/C and (e) N-HP-Co SACs at a current density of $100 \mathrm{~mA} \mathrm{~g}^{-1}$ with a limiting specific capacity of $3000 \mathrm{mAh} \mathrm{g}^{-1}, 500 \mathrm{~nm}$ in (d, e); $\mathbf{f}$ The corresponding XRD patterns. The standard spectra for $\mathrm{Li}_{2} \mathrm{O}_{2}$ are also shown for reference. Discharge mechanism of $0.6 \%$ commercial Pt/C $(\mathbf{g})$ and N-HP Co SACs $(\mathbf{h})$ in the $\mathrm{Li}_{-} \mathrm{O}_{2}$ batteries.

also measured under the same conditions. Figure 4 a shows that a low discharge voltage $(0.145 \mathrm{~V})$ can be achieved with the N-HPCo SACs. In addition, the ORR polarization curves (Supplementary Fig. 8) suggest that the kinetics of $\mathrm{Li}_{2} \mathrm{O}_{2}$ deposition are greatly improved by the special SACs, which is further supported by the CV curves in Fig. 3b. Compared with $0.6 \%$ commercial Pt/ $\mathrm{C}$ catalyst-loaded cathode, a higher onset potential and larger peak current density are obtained with N-HP-Co SAC catalysts for ORR, thus suggesting the enhanced activity on ORR with SACs. The superior ORR activity of the N-HP-Co SAC catalysts can be attributed to the following two reasons: first, the charge redistribution induced by the decoration of isolated Co atoms improves the $\mathrm{O}_{2}$ adsorption and reduction efficiency and second, the abundance of exposed active centres are provided by the special architecture, which guarantees facile electrocatalytic kinetics $^{34}$.
The superior ORR activity of N-HP-Co SACs further inspired us to explore the rate performance. As expected, the rate performance investigations (Fig. 4c) show that the discharge voltage plateau of the N-HP-Co SAC catalyst is higher than that of $0.6 \%$ commercial $\mathrm{Pt} / \mathrm{C}$ at each current density. The first full discharge curves (Supplementary Fig. 9) of the two catalysts and the other commonly used catalysts in $\mathrm{Li}-\mathrm{O}_{2}$ cells, along with the EIS results (Supplementary Fig. 10), are consistent with the above result, which indicates more efficient electron, ionic and mass transport. Such a large difference is attributed to the large number of exposed $\mathrm{CoN}_{4}$ sites on the surface of the N-HP-Co SACs, which is beneficial for absorbing oxygen molecules and promoting electron transfer.

Moreover, the morphology of $\mathrm{Li}_{2} \mathrm{O}_{2}$ can also be manipulated in situ by virtue of the surface properties of the Co SACs. Figure 4d, e show the SEM images of discharged cathodes with 
the $0.6 \%$ commercial Pt/C and N-HP-Co SAC catalysts. Notably, the morphology of the discharge products is dramatically different even with the same discharge current density of 100 $\mathrm{mA} \mathrm{g}^{-1}$. On the cathodes with the $0.6 \%$ commercial $\mathrm{Pt} / \mathrm{C}$ catalyst, the discharge products demonstrate a toroidal morphology on the carbon surface after discharge, which is consistent with the results obtained by other groups ${ }^{35,36}$. However, when the N-HP-Co SACs was used as the cathode, unique nanosheets uniformly grow on the wall of the porous carbon spheres. Such observations also directly indicate the strong interaction of the metal atoms with the supports and the dynamic structural transformation during the discharge process ${ }^{37}$. The PXRD patterns, FTIR and XPS spectra (Fig. 4f, Supplementary Figs. 11 and 12) for the carbon cathode after the discharge process also show that the main discharge product is $\mathrm{Li}_{2} \mathrm{O}_{2}$. Clearly, the nanosheet growth corresponds to the complex electronic structure at the $\mathrm{CoN}_{4}$ sites where the $\mathrm{Li}^{+}$, electrons and $\mathrm{O}_{2}$ meet and are subsequently fed to the surface as "seeds" and then to the bottom side as $\mathrm{Li}_{2} \mathrm{O}_{2}$. This indicates that the growth process is sustained by continuous mass transport to the top of the nanosheets, which is benefited by the directional alignment of the Co SACs. The functional schematic illustration of the catalysts in $\mathrm{Li}-\mathrm{O}_{2}$ batteries is shown in Fig. $4 \mathrm{~g}$, h. The increased discharge capacity of the N-HP-Co $\mathrm{SAC}$ is due to the increased $\mathrm{CoN}_{4}$ as "active seeds" in the Ndoped hollow carbon matrix. The $\mathrm{Li}_{2} \mathrm{O}_{2}$ nanoparticles are preferentially formed on the cathode, which can serve as nucleation sites for subsequent $\mathrm{Li}_{2} \mathrm{O}_{2}$ growth. Under the same current and capacity, the N-HP-Co SAC will provide more nucleation sites that are favourable for the formation of small nanosheets and the further self-assembly of large sheets according to the enhanced solvation-mediated mechanism ${ }^{36}$. Furthermore, a special discharge mechanism is in favour of the charge process, such as charge-transport limitations and the electronically insulating property of the discharge products.

Recharging behaviour with the N-HP-Co SAC catalysts. For the charging behaviour, Fig. 5 indicates that the charge voltage is much lower than that of the $0.6 \%$ commercial $\mathrm{Pt} / \mathrm{C}$ catalyst loaded on a CP cathode at $\sim 500 \mathrm{mV}$. To determine whether the improved electrochemical performance is caused by the Co SAC catalysts, the full discharge/charge analysis with the commonly used $0.6 \%$ catalyst is displayed in Supplementary Fig. 9. The single Co atom exhibits a lower OER overpotential compared with those of other metal catalysts in $\mathrm{Li}-\mathrm{O}_{2}$ batteries (Supplementary Table 4). Moreover, a 5\% commercial Pt/C was used for comparison (Supplementary Fig. 9a), and the N-HP-Co SACs exhibit comparable advantages in both the discharge and charge reactions. This fact is further verified by the CV curves (Fig. 4b); three obvious peaks occur during charging for the decomposition of $\mathrm{Li}_{2} \mathrm{O}_{2}$. First, the peak at $\sim 2.76 \mathrm{~V}$ may be related to $\mathrm{LiO}_{2}$ oxidation in the electrolyte ${ }^{38}$. The peak at $\sim 3.2 \mathrm{~V}$ in the anodic scan of the Co SAC catalysts originates from the suitable decomposition of nonstoichiometric $\mathrm{Li}_{2-x} \mathrm{O}_{2}$, especially outside of $\mathrm{Li}_{2} \mathrm{O}_{2}$. The third peak at $\sim 3.3 \mathrm{~V}$ can be assigned to the oxidation of $\mathrm{Li}_{2} \mathrm{O}_{2}$, which is mainly formed by the solvation-mediated discharge process through a one-electron transfer. However, only one broad weak peak at $4.31 \mathrm{~V}$ is observed in the $\mathrm{Pt} / \mathrm{C}$ electrode, thus demonstrating fewer chances for the dissociation of the formed $\mathrm{Li}_{2} \mathrm{O}_{2}$ followed by a two-electron transfer or other reaction products such as $\mathrm{LiOH}$ or $\mathrm{Li}_{2} \mathrm{CO}_{3}$ requiring high thermodynamic potentials ${ }^{39}$. These results indicate that the N-HP-Co SACs possess excellent functional activities towards the ORR/ OER, which are beneficial for improving the $\mathrm{Li}-\mathrm{O}_{2}$ battery performance. For the charging process, the peaks of $\mathrm{Li}_{2} \mathrm{O}_{2}$ disappear, and the XRD patterns (Supplementary Fig. 13) are the same as those of the pristine cathode before discharge, which is consistent with the impedance analysis results (Supplementary Fig. 10). It is generally accepted that the electrochemical decomposition of $\mathrm{Li}_{2} \mathrm{O}_{2}$ will follow these two steps: first, $\mathrm{Li}_{2} \mathrm{O}_{2} \rightarrow \mathrm{Li}^{+}+\mathrm{e}^{-}+\mathrm{LiO}_{2}$, and second, $\mathrm{LiO}_{2} \rightarrow \mathrm{Li}^{+}+\mathrm{e}^{-}+\mathrm{O}_{2}$. Simply, $\mathrm{Li}_{2} \mathrm{O}_{2}$ is first extracted from a $\mathrm{Li}$ atom to form a $\mathrm{LiO}_{2}$ intermediate. The $\mathrm{LiO}_{2}$ consequently releases oxygen through electrochemical or chemical reactions in the presence of effective catalysts. To verify the $\mathrm{Li}_{2} \mathrm{O}_{2}$ oxidation on the N-HP-Co SACs, UV-vis measurements were used to quantitatively monitor the $\mathrm{LiO}_{2}$ intermediates ${ }^{40,41}$. Based on the spectra obtained from the DMSO solution dissolved with a certain amount of $\mathrm{KO}_{2}$ (Supplementary Fig. 14), the absorption peak at $262 \mathrm{~nm}$ can be assigned to superoxide species. As shown in Fig. 5a, the spectra of the different charged electrodes have a similar absorption peak of the superoxide intermediates at $\sim 262 \mathrm{~nm}$, which confirms the two-step decomposition of $\mathrm{Li}_{2} \mathrm{O}_{2}$. More significantly, the UV-vis absorbance shows that the two types of cathodes have significant differences even at the same charge stage. Obviously, the absorbance values from the N-HP-Co SACs were all higher than those of the commercial $\mathrm{Pt} / \mathrm{C}$ catalyst at stages a and b (the state in Fig. 5a), which means that more $\mathrm{LiO}_{2}$ intermediates form during the charging process. Thus, the extraordinary SACs can bridge the gap between homogeneous and heterogeneous catalysis by virtue of the complex coordination environment around the active centres, quantum size effect and support effect ${ }^{42}$. Compared with heterogeneous catalysts, Co SACs exhibit a low metal loading but high atom utilization and homogeneous active $\mathrm{CoN}_{4}$ sites with tunable electronic environments towards $\mathrm{Li}_{2} \mathrm{O}_{2}$ oxidation for high catalytic activity through a one-electron reaction. As shown in Supplementary Figs. 15 and 16, more $\mathrm{O}_{2}{ }^{-}$existed in the electrolyte with N-HP-Co SACs according to the nitrotetrazolium blue chloride detection and EPR spectra ${ }^{40,43}$.

Based on the above results, the charging mechanism is presumed in Fig. 5b. At the relatively low charge potential of the Co SACs (Fig. 5a, Supplementary Fig. 9), the nanosheet-like $\mathrm{Li}_{2} \mathrm{O}_{2}$ may be the first to undergo a topotactic delithiation to form off-stoichiometric $\mathrm{Li}_{2-x} \mathrm{O}_{2}$. The $\mathrm{N}$-doped $s p^{2}$ carbon can improve the binding energies, further regulating the electron structure and the adsorption energy of the intermediate $\mathrm{LiO}_{2}$. Then, the increased $\mathrm{LiO}_{2}$ intermediates indicate that the recharge process will probably convert from a two-electron reaction to a singleelectron reaction. The main postulation based on the complex environment around single $\mathrm{CoN}_{4}$ sites is that they will interact preferentially with $\mathrm{LiO}_{2}$ based on the ever-changing electron densities, further modulating the electrochemical activity during charging. Density functional theory (DFT) calculations were further used to understand the possible effect of the cathode surface properties on the $\mathrm{Li}_{2} \mathrm{O}_{2}$ decomposition pathway. The optimized structures and the corresponding binding energies between the reduced species $\left(\mathrm{LiO}_{2}\right.$ ion pairs) on the $\mathrm{Pt} / \mathrm{C}((111)$, according to Supplementary Fig. 17), N-HP-CS, and N-HP-Co SAC surfaces are shown in Fig. $5 c, d, f$, and $g$ and Supplementary Fig. 18. We find that the binding energies of the reduced species on the Pt/C $(-1.85 \mathrm{eV})$ and N-HP-CSs $(-1.55 \mathrm{eV})$ surfaces are all much higher than those on the N-HP-Co SAC surface $(-1.03 \mathrm{eV})$, indicating that the "agravic" species more easily spread to the electrolyte from the N-HP-Co SAC surface. Therefore, the decomposition of $\mathrm{Li}_{2} \mathrm{O}_{2}$ is likely to be a oneelectron reaction, which is in good agreement with the experimental observations (Fig. 5a, Supplementary Figs. 15 and 16). Moreover, the corresponding charge density difference diagrams of the $\mathrm{Pt}(111)$ and $\mathrm{CoN}_{4}$ systems are shown in Fig. 5e, $\mathrm{h}$; the yellow area is the gained electron and the blue area is the lost electron, indicating that the reaction occurs on the $\mathrm{CoN}_{4}$ sites with an abundance of electron transfers compared with that on 
a

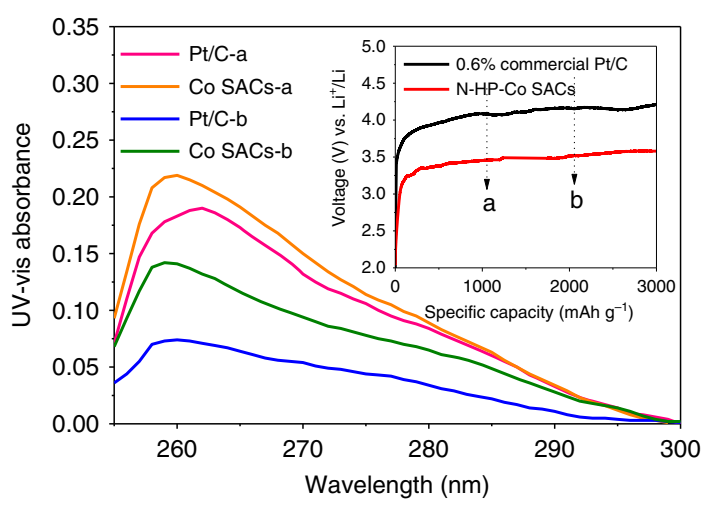

b

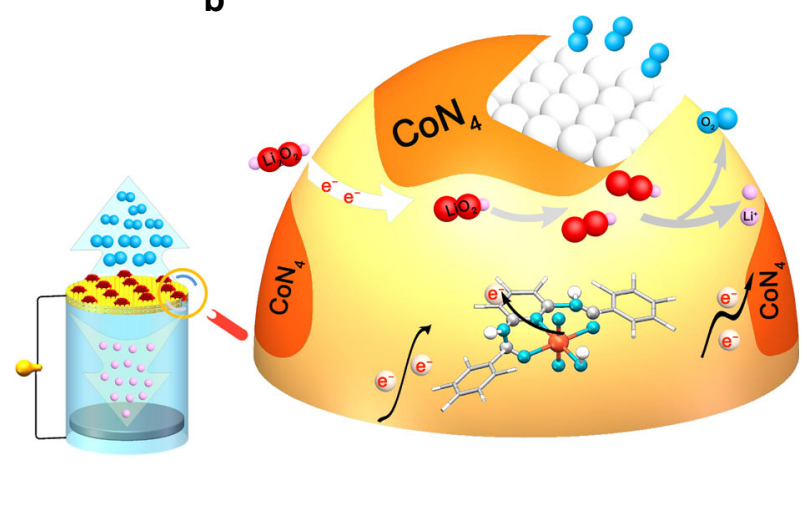

C

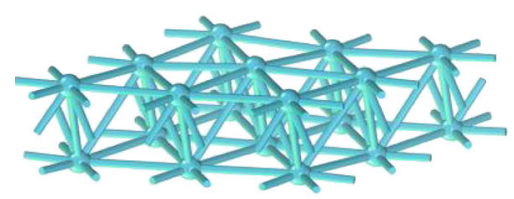

Pt (111)

$\mathbf{f}$

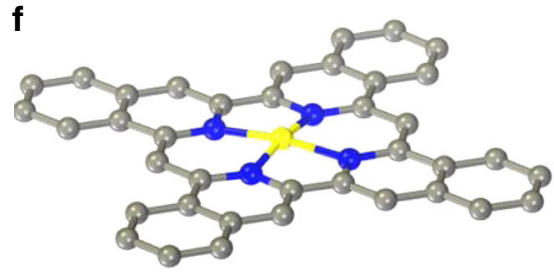

Pristine $\mathrm{CoN}_{4} \mathrm{SACs}$ d

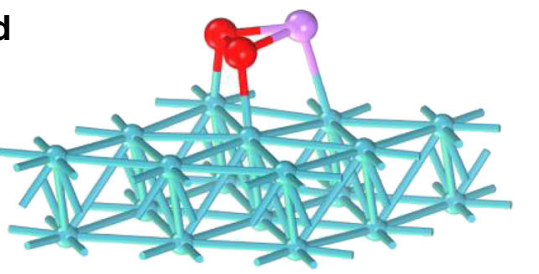

$\mathrm{LiO}_{2}$ on $\mathrm{Pt}$ (111)

$E_{\text {ads }}=-1.85 \mathrm{eV}$

g

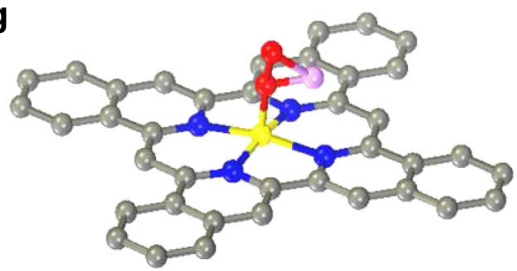

$\mathrm{LiO}_{2}$ on $\mathrm{CoN}_{4}$

$E_{\text {ads }}=-1.03 \mathrm{eV}$

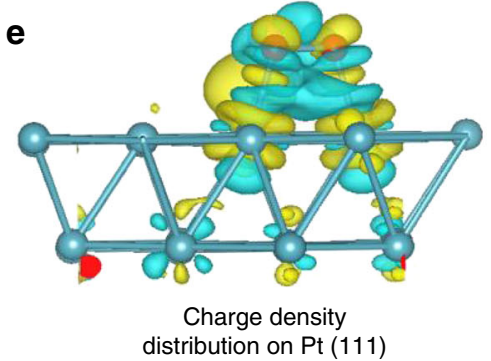

h

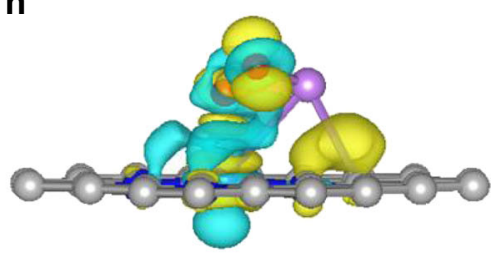

Charge density distribution on $\mathrm{CoN}_{4}$

Fig. 5 Charge characterization. a UV-vis spectra of DMSO-extracted superoxide products from the $0.6 \%$ commercial Pt/C and N-HP-Co SAC cathodes at different charging states (a state a, charge to $1000 \mathrm{mAh} \mathrm{g}^{-1}$; b state b, charge to $2000 \mathrm{mAh}^{-1}$ ). The inset of a shows the corresponding charge potential profiles of the $\mathrm{Li}-\mathrm{O}_{2}$ cells using $0.6 \%$ commercial Pt/C and N-HP-Co SACs at a current density of $100 \mathrm{~mA} \mathrm{~g}^{-1}$. b Schematic illustration showing the charge mechanisms of the N-HP-Co SAC-catalysed $\mathrm{Li}_{-} \mathrm{O}_{2}$ batteries. c, $\mathbf{d}$ Pristine and top views of the optimized structures with the corresponding binding energy of $\mathrm{LiO}_{2}$ on $\mathrm{Pt}(111) ; \mathbf{f}, \mathbf{g}$ optimized structure and the corresponding binding energy of $\mathrm{LiO}_{2}$ on $\mathrm{CoN}_{4}$; and $\mathbf{e}$, $\mathbf{h}$ corresponding charge density distribution. Colour code: platinum (green), carbon (grey), $\mathrm{CoN}_{4}$ (blue), and $\mathrm{LiO}_{2}$ (red).

the Pt sites. These results reveal that the single Co atom in the $\mathrm{Li}-\mathrm{O}_{2}$ batteries can form a more positive equilibrium system for the formation and consumption of $\mathrm{Li}_{2} \mathrm{O}_{2}$. However, we cannot ignore the effect of the discharge products on the OER because the shape and size of $\mathrm{Li}_{2} \mathrm{O}_{2}$ can significantly influence the charge potential profiles, which are further demonstrated by "chargeonly" reactions (see Supplementary Figs. 19, 20 and 21).

Stability of the electrochemistry in $\mathrm{Li}-\mathrm{O}_{2}$ batteries. We further examined the stability of the above reaction system with Co SACs. Figure 6a shows that the N-HP-Co SAC-loaded electrode exhibits stable discharge/recharge reactions for more than 250 cycles (218 days), whereas the $0.6 \%$ commercial $\mathrm{Pt} / \mathrm{C}$ electrode degrades after only 100 cycles when the terminal voltage reaches $2 \mathrm{~V}$. More importantly, the $\mathrm{Li}-\mathrm{O}_{2}$ cells with the N-HP-Co SACs still exhibit a good, stable specific capacity and retain a relatively stable terminal discharge voltage above $2 \mathrm{~V}$ after $\sim 50$ cycles at a large current density of $400 \mathrm{~mA} \mathrm{~g}^{-1}$, indicating excellent cycling stability. Figure $6 \mathrm{~b}, \mathrm{~d}$ shows the morphology of the discharged carbon electrodes after the 5 th and 20th cycles. In the presence of the $0.6 \%$ commercial $\mathrm{Pt} / \mathrm{C}$ catalyst, the particles with the pristine toroidal morphology disappear instead of the film-like discharge products. However, with the N-HP-Co SACs, only nanosheets, in a clear and compact manner, can be observed on the cathode surface after the 20th cycle (Fig. 6c, e). Importantly, the PXRD patterns (Supplementary Fig. 23) match well with the above results.

The degradation of the crystalline $\mathrm{Li}_{2} \mathrm{O}_{2}$ in cycling can be speculated to be the increasing accumulation of the side products on the surface of the cathode, which goes against the nucleation and crystallization of $\mathrm{Li}_{2} \mathrm{O}_{2}$ during the subsequent discharge, and leads to the formation of amorphous $\mathrm{Li}_{2} \mathrm{O}_{2}$. Figure $6 \mathrm{f}, \mathrm{g}$ and Supplementary Fig. 24 show the Li $1 s, \mathrm{O} 1 s$ and C $1 s$ spectra of the discharged cathodes after consecutive cycles. The Li $1 s$ and $\mathrm{O}$ $1 s$ peaks of $\mathrm{Li}_{2} \mathrm{O}_{2}$ at 55.04 and $531.62 \mathrm{eV}$, respectively, are consistent with previously reported XPS studies on $\mathrm{Li}_{2} \mathrm{O}_{2}$ formed in $\mathrm{Li}-\mathrm{O}_{2}$ cells ${ }^{44,45}$. However, as the cycle continues, the intensity obviously decreases, and a peak of $\mathrm{Li}_{2} \mathrm{CO}_{3}$ begins to appear on the cathodes of the $0.6 \%$ commercial $\mathrm{Pt} / \mathrm{C}$ catalyst. This result corresponds well with the changes in the $\mathrm{C} 1 \mathrm{~s}$ peaks (Supplementary Fig. 24); in detail, the C 1 s spectra of $0.6 \%$ commercial $\mathrm{Pt} / \mathrm{C}$ catalyst confirms the formation of a variety of parasitic C-containing products. For instance, the peaks at 288.7 
a

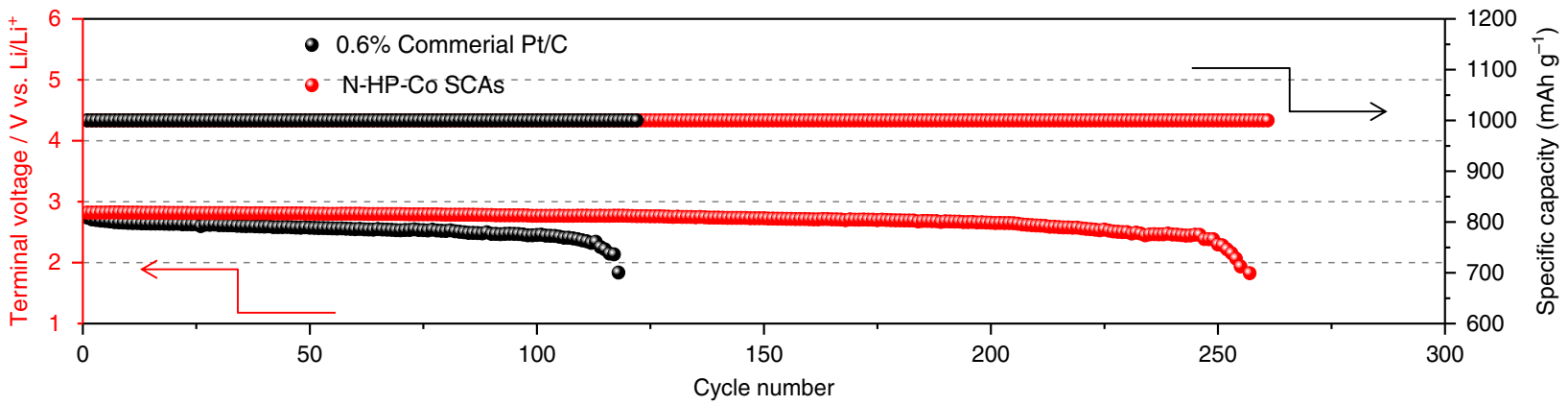

b

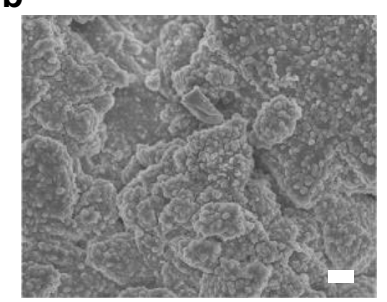

d

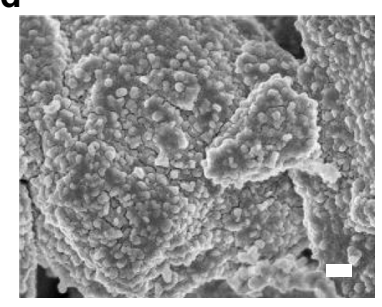

c

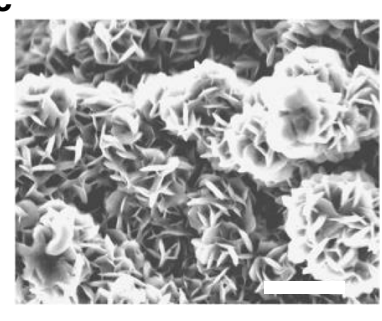

e

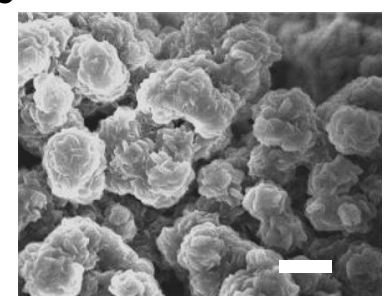

f

Li $1 \mathrm{~s}$

5th

$\mathrm{Li}_{2} \mathrm{CO}_{3} \mathrm{Li}_{2} \mathrm{O}_{2} \mathrm{LiOH}$
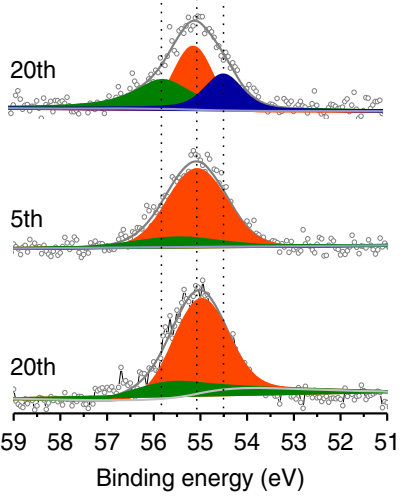

g

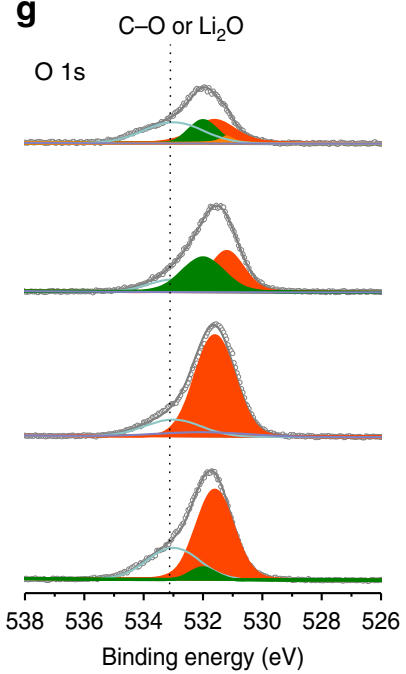

Fig. 6 Cycling stability of $\mathbf{L i}_{-} \mathbf{O}_{\mathbf{2}}$ batteries with $\mathbf{N}-\mathbf{H P}$-Co SACs. a Voltage versus cycle number on the discharge terminal of the Li- $\mathrm{O}_{2}$ cells with commercial Pt/C and N-HP-Co SACs. FESEM images of the discharged cathode with the $0.6 \%$ commercial Pt/C catalyst (b) and the N-HP-Co SAC catalyst (c) at a current density of $200 \mathrm{~mA} \mathrm{~g}^{-1}$ after the 5 th cycle and the cathode with the $0.6 \%$ commercial Pt/C catalyst (d) and the N-HP-Co SAC catalyst (e) after the 20th cycle. One micrometer in b, d; $500 \mathrm{~nm}$ in $\mathbf{c}, \mathbf{e} ; \mathbf{f}, \mathbf{g}$ XPS spectra of the discharged cathodes with $0.6 \%$ commercial Pt/C and N-HP-Co SACs: Li 1s (f) and O 1s (g) after the 5th and 20th cycles.

and $289.89 \mathrm{eV}$ exhibit the formation of a small amount of lithium carboxylates $\left(\mathrm{ROCO}_{2} \mathrm{Li}\right)$ and carbonates $\left(\mathrm{Li}_{2} \mathrm{CO}_{3}\right)$ upon the first discharge (Supplementary Fig. 12c). However, the amounts of these products increase with further cycling. In sharp contrast, minor peaks referring to by-products are found with the N-HPCo SAC-containing cathodes after the 20th cycle because of potential side reactions that occur between the discharge products and electrolyte solvent. These results are also confirmed by an FTIR analysis (Supplementary Fig. 25). Furthermore, the side products during the charging process were monitored by FTIR and NMR measurements, and the results are shown in Supplementary Figs. 26 and 27.

Based on the above results, the gas evolution during the charging reaction was monitored through an in situ differential electrochemical mass spectrometry analysis. As shown in Supplementary Fig. 28, the curves of $\mathrm{O}_{2}$ indicate that the main reaction is $\mathrm{Li}_{2} \mathrm{O}_{2}$ decomposition. Compared with N-HP-Co SACs, the $\mathrm{CO}_{2}$ pressure for $0.6 \%$ commercial $\mathrm{Pt} / \mathrm{C}$ is very large and increases with further cycling, which is possibly due to the side reactions on the carbon or the binder by a highly reactive radical. In addition, gas chromatography signals (Supplementary Fig. 29, Supplementary Table 3) were used to quantitatively analyze the reversibility of the reactions and indicated the $\mathrm{O}_{2}$ amount $(1.16 \mu \mathrm{L})$ of the N-HP-Co SACs after the first recharge. In addition, the difference can be illustrated by the $\mathrm{TiOSO}_{4}$-based quantitative results (Supplementary Fig. 30), in which the red bars are caused by the incomplete decomposition of $\mathrm{Li}_{2} \mathrm{O}_{2}{ }^{46}$. Specifically, the cell with the N-HP-Co SACs exhibits both a high $\mathrm{Li}_{2} \mathrm{O}_{2}$ formation efficiency (93.9\%) and low $\mathrm{Li}_{2} \mathrm{O}_{2}$ residual (4.8\%); specifically, the difference becomes increasingly clear with further cycling.

Durability of the N-HP-Co SACs after cycling in $\mathrm{Li}-\mathrm{O}_{2}$ batteries. In this regard, we conclude that the high surface energy of the Co SACs can protect the carbon support from the attack of the $\mathrm{O}^{2-}$ ion, thereby reducing the parasitic reactions on the carbon or binder. As shown in Fig. 7a, the XPS of the Co $2 p$ spectrum of the recharged cathode demonstrates that single $\mathrm{CoN}_{4}$ active sites still exist, and no Co-Co is observed. Because of the difficulty in observing the carbon support surface after 50 cycles, a relatively uniform distribution of $\mathrm{Co}$ and $\mathrm{N}$ is observed through EDX elemental mapping (Fig. 7b); the XAFS measurements (Fig. 7c, d) further demonstrate the single Co atom in the $\mathrm{N}$ doped carbon sphere support. This indicates that Co atoms are still bound to the carbon surface through a nitrogen-related ligand. However, as the cycling proceeds, the content of $\mathrm{Co}$ is unavoidably reduced. This is the next important problem that we need to overcome. In summary, the N-HP-Co SACs exhibit excellent activity, durability and stability in $\mathrm{Li}-\mathrm{O}_{2}$ batteries.

\section{Discussions}

In summary, inspired by the growth process of strawberries, a well-dispersed single Co atom catalyst was first prepared by a polymer encapsulation strategy with $\mathrm{a} \mathrm{SiO}_{2}$ template and then 
a

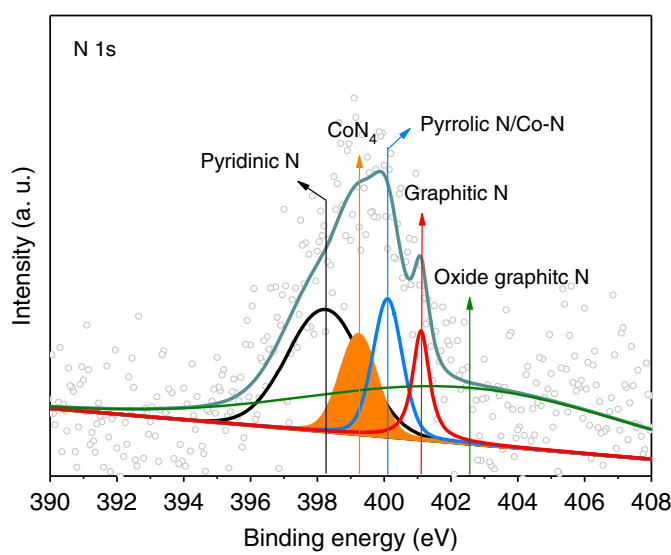

b

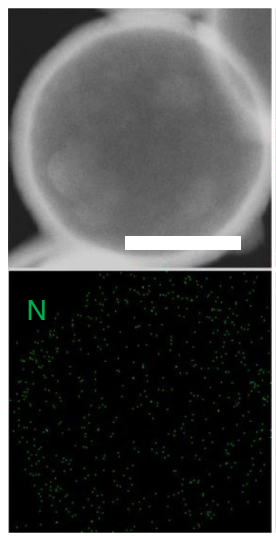

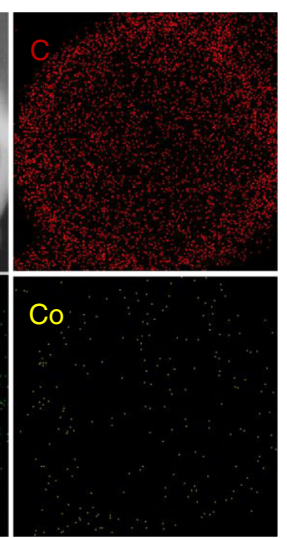
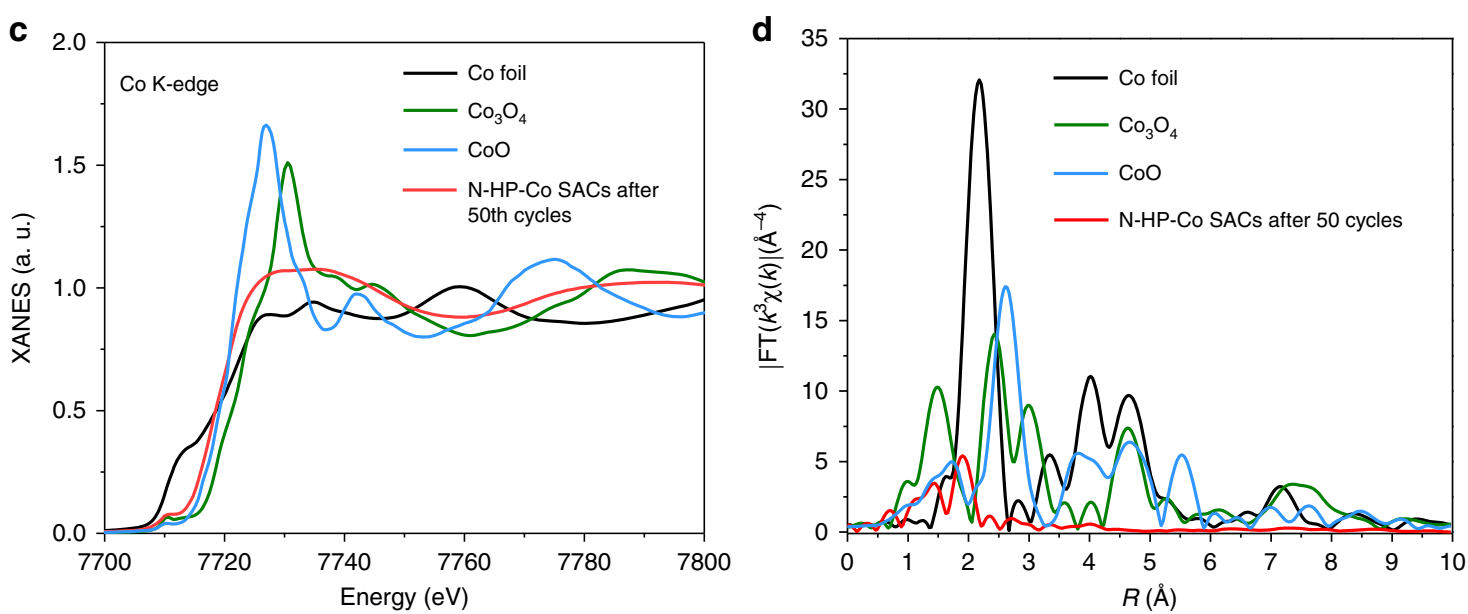

Fig. 7 Cycling stability of $\mathbf{N}$-HP-Co SACs. a N 1s XPS spectra for N-HP-Co SACs after 50 galvanostatic cycles. b HAADF-STEM images and the corresponding EDX elemental maps after 50 galvanostatic cycles, $200 \mathrm{~nm}$ in b. c, d XANES spectra and Fourier transform of the Co K-edge of N-HP-Co $\mathrm{SACs}, \mathrm{CO}_{3} \mathrm{O}_{4}, \mathrm{CoO}$, and $\mathrm{Co}$ foil.

pyrolysis under an inert atmosphere. The single-atom nature of the active centres in N-HP-Co SACs and the resulting lowcoordination environments and enhanced metal-support interactions provided remarkable catalytic performance and successfully regulated the deposition/decomposition routes in $\mathrm{Li}-\mathrm{O}_{2}$ batteries. Compared with commercial $\mathrm{Pt} / \mathrm{C}$ catalysts, the highly reactive activity Co SACs could attack more $\mathrm{O}^{2-}$, which not only accurately manipulated the discharge product but also increased the discharge capacity, thus contributing greatly to alleviating cathode passivation and side reactions. As a result, the Co SACcatalysed $\mathrm{Li}-\mathrm{O}_{2}$ batteries showed an ultralong cycle life (261 cycles at a current density of $100 \mathrm{~mA} \mathrm{~g}^{-1}$ with a cut off capacity of $\left.1000 \mathrm{mAh} \mathrm{g}^{-1}\right)$ and a high discharge capacity $(\sim 14,777 \mathrm{mAh} \mathrm{g}$ ${ }^{-1}$ at a current density of $100 \mathrm{~mA} \mathrm{~g}^{-1}$ ). For the charging process, the initial delithiation of $\mathrm{Li}_{2} \mathrm{O}_{2}$ was a one-electron process rather than the general two-electron mechanism, which was achieved by the dispersed single Co atom catalysts in the $\mathrm{Li}-\mathrm{O}_{2}$ cells. The oneelectron process is more kinetically favourable and highly reversible, leading to identical charging behaviour with noble-based catalysts. This work opens a new approach for the rational design of highly efficient noble metal-free electrocatalysts for energy storage and conversion applications.

\section{Methods}

Li- $\mathrm{O}_{2}$ cell preparation and electrochemical measurements. The electrochemical performance of the $\mathrm{Li}-\mathrm{O}_{2}$ cell was tested in a 2025-type coin cell. All of the cells were assembled in a glove box in an Ar atmosphere with a lithium foil anode, a glass fibre separator, an oxygen cathode and an electrolyte containing $1 \mathrm{M}$ LiTFSI in TEGDME. A pristine cathode was prepared by coating a homogenous ink, which was composed of a mixture of $80 \mathrm{wt} \% \mathrm{~N}-\mathrm{HP}-\mathrm{Co}$ SACs and $20 \mathrm{wt} \%$ poly(vinylidene fluoride), onto a CP current collector. In addition, a mixture of $80 \mathrm{wt} \%$ Super $\mathrm{P}$ containing $0.6 \%$ commercial $\mathrm{Pt} / \mathrm{C}$ catalyst and $20 \mathrm{wt} \%$ poly(vinylidene fluoride) was deposited on $\mathrm{CP}$ and prepared in the same manner. The active material loading was $\sim 0.35 \mathrm{mg} \mathrm{cm}^{-2}$. The electrochemical performances of the cathodes with N-HP-Co SAC and commercial Pt/C catalysts were tested in a specific capacity-controlled mode under various current densities. The electrochemical impedance spectroscopy of the cell was evaluated using a CHI660E electrochemical workstation (Shanghai chenhua instrument co., Ltd) in a frequency range of $10^{5}-10^{-2} \mathrm{~Hz}$.

Synthesis of N-HP-Co SACs by a polymer encapsulation approach. Silica spheres were used as templates to prepare hollow carbon spheres. In a typical experiment, $\mathrm{SiO}_{2}(0.15 \mathrm{~g})$, dopamine $(0.15 \mathrm{~g})$ and cobalt acetylacetonate $(3.6 \mathrm{mg})$ were dissolved in $100 \mathrm{~mL}$ deionized water and stirred for $10 \mathrm{~min}$. Tris-buffer $(1.21 \mathrm{~g})$ was added to adjust the $\mathrm{pH}$ of the resulting solvent to 8.5 and stirred for 12 h. The resulting Co(acac $)_{2} @ S_{2} \mathrm{~S}_{2} @ P D A$ nanospheres were suction filtrated and washed with distilled water several times. The N-HP-Co SACs were obtained by pyrolyzing $\mathrm{Co}(\mathrm{acac})_{2} @ \mathrm{SiO}_{2} @ \mathrm{PDA}$ at $900{ }^{\circ} \mathrm{C}$ in a $\mathrm{N}_{2}$ atmosphere and etching the silica core with HF.

Characterizations. The morphology and structure of the materials were characterized using various physicochemical techniques, including XRD, HAADFSTEM, XAFS measurements, field emission scanning electron microscopy (FESEM), TEM and XPS. The discharge and recharge products were characterized using XRD, SEM, NMR, FTIR, XPS, and UV-vis absorption spectrum technology. HAADF-STEM images and corresponding EDX elemental maps were characterized by using a JEM-ARM300F Grand ARM atomic resolution electron microscope with double Cs correctors. TEM was carried out with a JEM-2100F transmission electron microscope.

Theoretical calculations. All electronic structure and energy calculations were performed by spin-polarized DFT using the Vienna ab initio simulation package ${ }^{47-50}$ 
The projector-augmented wave (PAW) potentials were used to describe ion core and valence electron interactions ${ }^{51,52}$. A generalized gradient approximation with the Perdew-Burke-Ernzerhof functional ${ }^{53}$ was selected to describe the exchangecorrelation functional. A kinetic energy cut off of $400 \mathrm{eV}$ was used with a plane-wave basis set. The integration of the Brillouin zone was conducted using a $3 \times 3 \times 1$ Monkhorst-Pack grid ${ }^{54}$. The convergences of the force and the total energy were set as $0.05 \mathrm{eV}^{-1}$ and $1.0 \times 10^{-4} \mathrm{eV}$ atom ${ }^{-1}$, respectively. Van der Waals (VDW) interaction was employed in our calculations by the Rugers-Chalmers Van der Waals Density Functional (VDW-DF) approach ${ }^{55,56}$. The Pt (111) surface was obtained by cutting a Pt crystal along the [111] direction, and a $3 \times 3$ unit cell with three layers $\left(\mathrm{Pt}_{36}\right)$ was chosen. The atoms in the bottom two layers were fixed in their bulk positions, and those in the other layers were allowed to relax. The $\mathrm{CoN}_{4}$ and the different types of $\mathrm{N}$-doped carbon supports were prepared by a $5 \times 5$ supercell single layer graphene. A large distance of $15 \AA$ was selected to avoid an imaging interaction. To study the stability of $\mathrm{LiO}_{2}$ on the selected materials, the adsorption energy was defined as the following:

$$
E_{\mathrm{a}}=E_{\mathrm{LiO}_{2}}+E_{\mathrm{X}}-E_{\mathrm{LiO}_{2} @ \mathrm{X}}\left(\mathrm{X}=\mathrm{CoN}_{4}, \mathrm{Pt} 111, \mathrm{~N}-\text { doped carbon }\right),
$$

where $E_{\mathrm{LiO}_{2} @ \mathrm{X}}, E_{\mathrm{X}}, E_{\mathrm{LiO}_{2}}$ correspond to the total energies of the $\mathrm{LiO}_{2}$ and substrates, an isolated substrate and $\mathrm{LiO}_{2}$, respectively. A positive value suggests strong binding and stable chemisorption.

\section{Data availability}

The data supporting the findings of this study are available within the paper and it Supplementary Information, and from the corresponding author upon reasonable request.

Received: 7 October 2019; Accepted: 22 March 2020;

Published online: 04 May 2020

\section{References}

1. Xia, C., Kwok, C. Y. \& Nazar, L. F. A high-energy-density lithium-oxygen battery based on a reversible four-electron conversion to lithium oxide. Science 361, 777-781 (2018).

2. Liu, T. et al. Cycling $\mathrm{Li}-\mathrm{O}_{2}$ batteries via $\mathrm{LiOH}$ formation and decomposition. Science 35, 530-533 (2015).

3. Liu, Q. C. et al. In situ construction of stable tissue-directed/reinforced bifunctional separator/protection film on lithium anode for lithium-oxygen batteries. Adv. Mater. 27, 1606552 (2015).

4. McCloskey, B. D. et al. On the efficacy of electrocatalysis in nonaqueous $\mathrm{Li}_{-} \mathrm{O}_{2}$ batteries. J. Am. Chem. Soc. 133, 18038 (2011).

5. $\mathrm{Xu}, \mathrm{J}$. J. et al. Cathode surface-induced, solvation-mediated, micrometer-sized $\mathrm{Li}_{2} \mathrm{O}_{2}$ cycling for $\mathrm{Li}-\mathrm{O}_{2}$ batteries. Adv. Mater. 28, 9620-9628 (2016).

6. Zhang, T. \& Zhou, H. S. A reversible long-life lithium-air battery in ambient air. Nat. Commun. 4, 1817 (2013).

7. Zhang, H. B. et al. Active sites implanted carbon cages in core-shell architecture: highly active and durable electrocatalyst for hydrogen evolution reaction. ACS Nano 10, 684-694 (2016).

8. Yang, L., Zeng, X. F., Wang, W. C. \& Cao, D. P. Recent progress in MOFderived, heteroatom-doped porous carbons as highly efficient electrocatalysts for oxygen reduction reaction in fuel cells. Adv. Funct. Mater. 28, 1704537 (2018).

9. Qiu, Y. C. et al. High-Rate, Ultralong cycle-life lithium/lulfur batteries enabled by nitrogen-doped graphene. Nano Lett. 14, 4821-4827 (2014).

10. Zhang, J. K. et al. Three-dimensional graphene- $\mathrm{Co}_{3} \mathrm{O}_{4}$ cathodes for rechargeable $\mathrm{Li}-\mathrm{O}_{2}$ batteries. J. Mater. Chem. A 3, 1504-1510 (2015).

11. Wang, Z. C. et al. Phosphorus-doped $\mathrm{Co}_{3} \mathrm{O}_{4}$ nanowire array: a highly efficient bifunctional electrocatalyst for overall water splitting. ACS Catal. 8, 2236-2241 (2018).

12. Tong, $\mathrm{S}$. F. et al. Mesoporous $\mathrm{NiO}$ with a single-crystalline structure utilized as a noble metal-free catalyst for nonaqueous $\mathrm{Li}-\mathrm{O}_{2}$ batteries. J. Mater. Chem. A 3, 16177-16182 (2015).

13. Liu, Y. et al. Constructing patch-Ni-shelled Pt@Ni nanoparticles within confined nanoreactors for catalytic oxidation of insoluble polysulfides in Li-S batteries. Small 15, 1902431 (2019).

14. Lu, Y. C. et al. Platinum-gold nanoparticles: a highly active bifunctional electrocatalyst for rechargeable lithium-air batteries. J. Am. Chem. Soc. 132, 12170-12171 (2010)

15. Leng, L. M. et al. Enhancing the cyclability of $\mathrm{Li}-\mathrm{O}_{2}$ batteries using $\mathrm{PdM}$ alloy nanoparticles anchored on nitrogen-doped reduced graphene as the cathode catalyst. J. Power Sources 337, 173-179 (2017).

16. Xu, J. J., Wang, Z. L., Xu, D., Meng, F. Z. \& Zhang, X. B. 3D ordered macroporous $\mathrm{LaFeO}_{3}$ as efficient electrocatalyst for $\mathrm{Li}-\mathrm{O}_{2}$ batteries with enhanced rate capability and cyclic performance. Energy Environ. Sci. 7, 2213-2219 (2014)

17. Oh, S. H., Black, R., Pomerantseva, E., Lee, J. H. \& Nazar, L. F. Synthesis of a metallic mesoporous pyrochlore as a catalyst for lithium- $\mathrm{O}_{2}$ batteries. Nat. Chem. 4, 1004-1010 (2012).

18. Qiu, T., Tu, B. T., Saldana-Greco, D. \& Rappe, A. M. Ab initio simulation explains the enhancement of catalytic oxygen evolution on $\mathrm{CaMnO}_{3}$. ACS Catal. 8, 2218-2224 (2018).

19. Chen, Y. H., Freunberger, S. A., Peng, Z. Q., Barde, F. \& Bruce, P. G. Li- $\mathrm{O}_{2}$ battery with a dimethylformamide electrolyte. J. Am. Chem. Soc. 134, 7952-77957 (2012).

20. Lu, J. et al. A. Effect of the size-selective silver clusters on lithium peroxide morphology in lithium-oxygen batteries. Nat. Commun. 5, 4895 (2014).

21. Yao, W. T. et al. Tuning $\mathrm{Li}_{2} \mathrm{O}_{2}$ formation routes by facet engineering of $\mathrm{MnO}_{2}$ cathode catalysts. J. Am. Chem. Soc. 141, 12832-12838 (2019).

22. Zhang, Z. Q. et al. The simplest construction of single-site catalysts by the synergism of micropore trapping and nitrogen anchoring. Nat. Commun. 10, 1657 (2019).

23. Cao, L. et al. Identification of single-atom active sites in carbon-based cobalt catalysts during electrocatalytic hydrogen evolution. Nat. Catal. 2, 134-141 (2019).

24. Gu, J., Hsu, C.-S., Bai, L. C., Chen, H. M. \& Hu, X. L. Atomically dispersed $\mathrm{Fe}^{3}$ + sites catalyze efficient $\mathrm{CO}_{2}$ electroreduction to CO. Science 364, 1091-1094 (2019).

25. Gao, C. et al. Heterogeneous single-atom catalyst for visible-light driven highturnover $\mathrm{CO}_{2}$ reduction: the role of electron transfer. Adv. Mater. 30, 1704624 (2018).

26. Liu, D. B. et al. Atomically dispersed platinum supported on curved carbon supports for efficient electrocatalytic hydrogen evolution. Nat. Energy 4, 512-518 (2019).

27. Fei, H. L. et al. Atomic cobalt on nitrogen-doped graphene for hydrogen generation. Nat. Commun. 6, 8668 (2015).

28. Lee, B. H. et al. Reversible and cooperative photoactivation of single-atom $\mathrm{Cu} /$ $\mathrm{TiO}_{2}$ photocatalysts. Nat. Mater. 18, 620-626 (2019).

29. Li, X. G. et al. Single-atom Pt as Co-catalyst for enhanced photocatalytic $\mathrm{H}_{2}$ evolution. Adv. Mater. 28, 2427-2431 (2016).

30. Stber, W., Fink, A. \& Bohn, E. Controlled growth of monodisperse silica spheres in the micron size range. J. Colloid Interface Sci. 26, 62-69 (1968).

31. He, Y. H. et al. Highly active atomically dispersed $\mathrm{CoN}_{4}$ fuel cell cathode catalysts derived from surfactant-assisted MOFs: carbon-shell confinement strategy. Energy Environ. Sci. 12, 250-260 (2019).

32. Zang, W. J. et al. Single Co atoms anchored in porous N-doped carbon for efficient zinc-air battery cathodes. ACS Catal. 8, 8961-8969 (2018).

33. Ai, K., Liu, Y. L., Ruan, C. P., Lu, L. H. \& Lu, G. Q. (Max) Sp ${ }^{2}$ C-dominant Ndoped carbon sub-micrometer spheres with a tunable size: a versatile platform for highly efficient oxygen-reduction catalysts. Adv. Mater. 25, 998-1003 (2013).

34. Zhang, H. B. et al. A modular strategy for decorating isolated cobalt atoms into multichannel carbon matrix for electrocatalytic oxygen reduction. Energy Environ. Sci. 11, 1980-1984 (2018).

35. Song, K. et al. Anisotropic surface modulation of Pt catalysts for highly reversible Li- $\mathrm{O}_{2}$ batteries: high index facet as a critical descriptor. ACS Catal. 8, 9006-9015 (2018).

36. Wu, F. et al. Platinum-coated hollow graphene nanocages as cathode used in lithium-oxygen batteries. Adv. Funct. Mater. 26, 7626-7633 (2016).

37. Yang, Y. et al. Tuning the morphology and crystal structure of $\mathrm{Li}_{2} \mathrm{O}_{2}:$ a graphene model electrode study for $\mathrm{Li}-\mathrm{O}_{2}$ battery. ACS Appl. Mater. Interfaces 8, 21350-21357 (2016).

38. Shen, Z. Z. et al. Revealing the surface effect of the soluble catalyst on oxygen reduction/evolution in $\mathrm{Li}-\mathrm{O}_{2}$ batteries. J. Am. Chem. Soc. 141, 6900-6905 (2019).

39. Yoon, K. R. et al. Brush-Llike cobalt nitride anchored carbon nanofiber membrane: current collector catalyst integrated cathode for long cycle $\mathrm{Li}-\mathrm{O}_{2}$ batteries. ACS Nano 12, 128-139 (2018).

40. Lin, X. D. et al. Controlling reversible expansion of $\mathrm{Li}_{2} \mathrm{O}_{2}$ formation and decomposition by modifying electrolyte in $\mathrm{Li}_{-} \mathrm{O}_{2}$ batteries. Chemistry $4,1-14$ (2018).

41. Cai, S. et al. A synergistic catalytic mechanism for oxygen evolution reaction in aprotic $\mathrm{Li}-\mathrm{O}_{2}$ battery. ACS Catal. 8, 7983-7990 (2018).

42. Yang, X.-F. et al. Single-atom catalysts: a new frontier in heterogeneous catalysis. Acc. Chem. Res. 46, 1740-1748 (2013).

43. Xie, J. et al. Achieving low overpotential $\mathrm{Li}-\mathrm{O}_{2}$ battery operations by $\mathrm{Li}_{2} \mathrm{O}_{2}$ decomposition through one-electron processes. Nano Lett. 15, 8371-8376 (2015).

44. Younesi, R., Hahlin, M., Björefors, F., Johansson, P. \& Edström, K. Li- $\mathrm{O}_{2}$ battery degradation by lithium peroxide $\left(\mathrm{Li}_{2} \mathrm{O}_{2}\right)$ : a model study. Chem. Mater. 25, 77-84 (2013).

45. Lu, Y. C. et al. In situ ambient pressure X-ray photoelectron spectroscopy studies of lithium-oxygen redox reactions. Sci. Rep. 2, 715 (2012). 
46. Qiao, Y. et al. MOF-based separator in an $\mathrm{Li}-\mathrm{O}_{2}$ battery: an effective strategy to restrain the shuttling of dual redox mediators. ACS Energy Lett. 3, 463-468 (2018).

47. Kresse, G. \& Furthmuller, J. Efficiency of ab-initio total energy calculations for metals and semiconductors using a plane-wave basis set. Comp. Mater. Sci. 6, 15-50 (1996).

48. Kresse, G. \& Hafner, J. Ab initio molecular dynamics for liquid metals. Phys. Rev. B 47, 558-560 (1993).

49. Kresse, G. \& Hafner, J. Ab initio molecular-dynamics simulation of the liquidmetal-amorphous-semiconductor transition in germanium. Phys. Rev. B 49, 14251-14269 (1994).

50. Kresse, G. \& Furthmuller, J. Efficient iterative schemes for ab initio totalenergy calculations using a plane-wave basis set. Phys. Rev. B 54, 11169-11186 (1996).

51. Blochl, P. E. Projector augmented-wave method. Phys. Rev. B 50, 17953-17979 (1994).

52. Kresse, G. \& Joubert, D. From ultrasoft pseudopotentials to the projector augmented-wave method. Phys. Rev. B 59, 1758-1775 (1999)

53. Perdew, J. P., Burke, K. \& Ernzerhof, M. Generalized gradient approximation made simple. Phys. Rev. Lett. 77, 3865-3868 (1996).

54. Monkhorst, H. J. \& Pack, J. D. Special points for Brillouin-zone integrations. Phys. Rev. B 13, 5188-5192 (1976).

55. Berland, K. et al. Van der waals forces in density functional theory: a review of the vdW-DF method. Rep. Prog. Phys. 78, 066501 (2015).

56. Thonhauser, T. et al. Spin signature of nonlocal correlation binding in metalorganic frameworks. Phys. Rev. Lett. 115, 136402 (2015).

\section{Acknowledgements}

This work was financially supported by the National Natural Science Foundation of China (Grant No. 51771177, 51972141), the 111 Project (B17020), the Education Department of Jilin Province (JJKH20190113KJ), Jilin Province Science and Technology Development Program (Grant No. 20190303104SF) and the Jilin Province/Jilin University Co-construction Project-Funds for New Materials (SXGJSF2017-3).

\section{Author contributions}

J.J.X. and L.N.S. developed the concept, designed the experiments. W.Z. and X.G. per formed and analyzed the high-angle annular dark field scanning transmission electron microscopy (HAADF-STEM) tests. Y.W was responsible for the theoretical computations. L.C.Z, H.F.W, X.X.W, Q.C.L, and F.L were all involved in discussions. J.J.X., L.N.S., W.Z., and Y.W wrote the paper. All of the authors discussed the results and reviewed the manuscript.

\section{Competing interests}

The authors declare no competing interests.

\section{Additional information}

Supplementary information is available for this paper at https://doi.org/10.1038/s41467020-15712-z.

Correspondence and requests for materials should be addressed to J.-J.X.

Peer review information Nature Communications thanks the anonymous reviewer(s) for their contribution to the peer review of this work. Peer reviewer reports are available.

Reprints and permission information is available at http://www.nature.com/reprints

Publisher's note Springer Nature remains neutral with regard to jurisdictional claims in published maps and institutional affiliations.

cc (i) Open Access This article is licensed under a Creative Commons Attribution 4.0 International License, which permits use, sharing, adaptation, distribution and reproduction in any medium or format, as long as you give appropriate credit to the original author(s) and the source, provide a link to the Creative Commons license, and indicate if changes were made. The images or other third party material in this article are included in the article's Creative Commons license, unles indicated otherwise in a credit line to the material. If material is not included in the article's Creative Commons license and your intended use is not permitted by statutory regulation or exceeds the permitted use, you will need to obtain permission directly from the copyright holder. To view a copy of this license, visit http://creativecommons.org/ licenses/by/4.0\%

(c) The Author(s) 2020 